\title{
Study on Chinese Spatial Metaphors at Lexical Level
}

\author{
Jihua Fan \\ School of Foreign Languages, North China Institute of Science and Technology, Beijing, China
}

\begin{abstract}
Study of spatial metaphors is a major theme in cognitive linguistics. This paper tries to make a full survey on Chinese spatial terms that are metaphorically extended to other domains and make a simple study on how they are metaphorically extended, with the aim of supporting and completing the contemporary cognitive theory of metaphor. Firstly this paper investigates the research state at present, then it briefly discusses the expressing form of spatial concepts in modern Chinese language, this defines the scope where our study can find the metaphorical extension of spatial concepts at linguistic level. At the same time, we still argue that some of them emerge directly from our spatial experience and some are metaphorically derived. There still exist the spatial expressions that are combined by body-part nouns plus spatial terms, which are also metaphorically used in Chinese expressions. Through these analyses, we can find some implication to the development of Chinese lexis.
\end{abstract}

Index Terms - spatial metaphor, Chinese lexis, spatial concepts

\section{INTRODUCTION}

Over past two decades since Lakoff and Johnson (1980) discussed the spatial metaphors, a number of studies have been made about this aspect. Such studies talk much on the linguistic phenomena occurring in various languages and try to explain them from a cognitive viewpoint. However, attention paid to this field is far from enough. As we move through the world, new visual, auditory, sensory inputs are continuously presented to the brain. We use such sensory and motor information to construct an internal representation of the space we perceive. The nature of this representation and the neural mechanisms underlying it has become a topic of great interest on cognitive neuroscience (Colby, 2000). Spatial perception allows us to achieve the concept of space directly through our bodily experience. Therefore, such basic concept is inevitably used in other expressions. Study of this linguistic phenomenon can throw some light on human cognition.

Space exists in the objective world. People living in such a world, can sense it through their interaction with the world as well as to express it through their languages. For the differences existing in the languages, the expressions in deferent languages may have some difference. To make this point clear, we take comparison between western languages and Chinese language.

\section{StUdies On Spatial Metaphors}

\section{A. Western Studies on Spatial Metaphor}

For spatial metaphors, Lakoff and Johnson (1980) have talked more by using English expressions. They say human beings build up a great number of spatial concepts: such as up/down, high/low, front/back, large/small etc. They claim such spatial terms are metaphorically extended to other concepts. What they claim is supported by many scholars (e.g. Kövecses 1995, 2000a, 2000b, Li 1995, Yu 1995, Alverson 1994, Shinohara 1998, etc.).

Lakoff and Johnson (1980) take spatial metaphors as one of their main classifications. They use a good number of linguistic expressions in English to analyze the extension of spatial terms used in other concepts. They loosely define such kind of metaphors as "that organizes a whole system of concepts with respect to one another...since most of them have to do with spatial orientation: up-down, in-out, front-back, on-off, deep-shallow, central-peripheral". But Lakoff and Johnson just simply exemplify spatial metaphors linked with spatial terms in English to verify their conceptual metaphor theory.

As we know, spatial metaphors belong to conventional ones, which we hardly recognize as metaphors when they are used in our daily life. For example, when we say "before 6 o'clock" "after 10 a.m.", we hardly sense they are metaphorically used here at all. However, such metaphors are governed by our experience as well as our culture customs. People with different culture customs may have different interpretations to space. For example, some regard "past time" as "front", "future time" as "back". While others think this in a contrary way: "past time" as "back", "future time" as "front".

The major studies of spatial metaphor lay on the representations of time and emotion. Spatial terms used in time are studied not only in English but also in other languages (Nuñez and Sweeter 2001, Yu 1995, Li 1995, Alverson, 1994). 
Another extension of spatial terms is emotion, that is to say, spatial terms are metaphorically used in emotional expressions. For instance, the metaphor HAPPY IS UP can be embodied in the following linguistic expressions:

(1) a. I'm feeling up.

b. You're in high spirit

c. That boosted my spirits.

d. My spirits rose.

e. You're in high spirits.

For this spatial metaphor, Lakoff and Johnson (1980) also explain the physical basis to form it: Drooping posture typically goes along with sadness and depression, erect posture with a positive emotional state. On this aspect, Kövecses (1995, 2000a, 2000b) has done much research. Apart from time and emotion, spatial terms also expand to other abstract concepts such as CONTROL, STATUS, and VIRTUE etc.

Based on the above discussion, we can conclude that these studies mainly focus on up/down and front/back, which are metaphorically extended to emotion and time, we can illustrate this in the following figure:

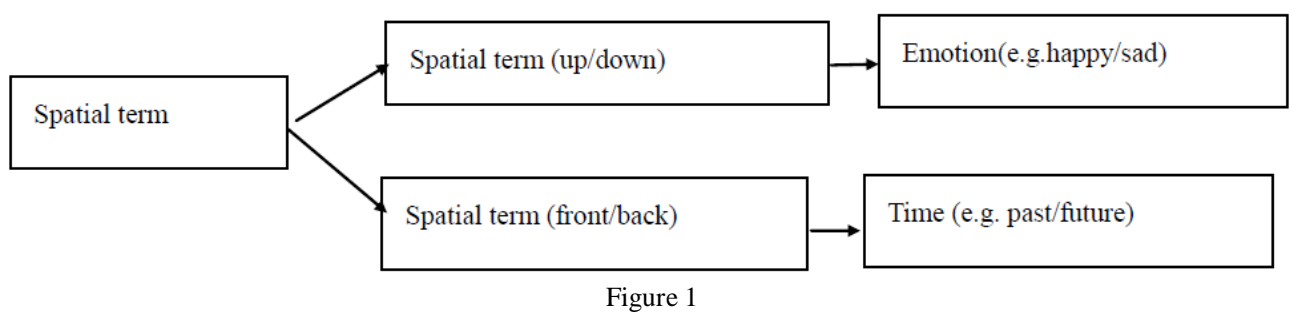

However, these studies just discuss some of the spatial terms from a cognitive prospect and support Lakoffian empiricalism. There still exists a lack of detailed study on spatial terms and their metaphorical extension in language, from which we can have a systematic look at the metaphorical extension of a set basic concept, in order to achieve some enlightenment, and provide a persuadable explaining theory and an able analyzing method to describe the working mechanism of metaphor, and, thus, to complete the cognitive theory of metaphor.

\section{B. Studies on Chinese Spatial Metaphors}

Integrating modern theories of metaphor, many scholars have done research on spatial metaphors by using real-life data in Chinese language, such as Lan (1999), Zhou (2002), and etc. But Lan only discusses the use of Chinese spatial terms shang/xia (up/down). Zhou only talks about the spatial terms qian/hou (front/back) used in the concept of time. From the aspect of a comparative linguistics, Yu (1995), Li (1995) and Alverson (1994) have also studied Chinese spatial metaphors. They have found quantities of linguistic evidences to support cognitive theory of metaphor. But they don't systematically study how Chinese spatial terms are metaphorically extended to other concepts. These studies are just based on the research of Lakoff and Johnson's and focus on spatial terms up/down and front/back. They don't discuss other spatial terms metaphorically used in Chinese language. They don't do further research in this field so as to find an effective way to explain metaphor. Even some of them give incomplete interpretations to Chinese spatial metaphors. For instance, Lan's explanation of why "up/down" use in Chinese time representations cannot work for other linguistic expressions such as zao shang (early up, which means early morning) and wan shang (late up, which means evening). Alverson (1994) only gives one sole conceptualization of time in Chinese. This is not true to Chinese spatial metaphors used in time. For this, we will further discuss in next paper.

On the other hand, some of the Chinese scholars (e.g., Huang 2001, Hu 2001), especially those with Chinese background, that is, those who had majored in the Chinese language and whose main concern is also in this language, are suspicious of the new theories that are being constantly generated by their western counterparts. They still adhere to traditional rhetoric to study metaphor. So their studies still linger at the linguistic level. In this situation, it is necessary for us to apply the new western linguistic theories to the research on Chinese language. In doing so, we can also contribute to a general theory of language, because our new findings with our mother tongue will enrich the borrowed theories. It will also help us to have a better knowledge of the characteristics of our mother tongue.

Based on the above disscussion, we can conclude that studies on Chinese spatial metaphors are mainly divided into two groups, we can illustrate this in the following figure:

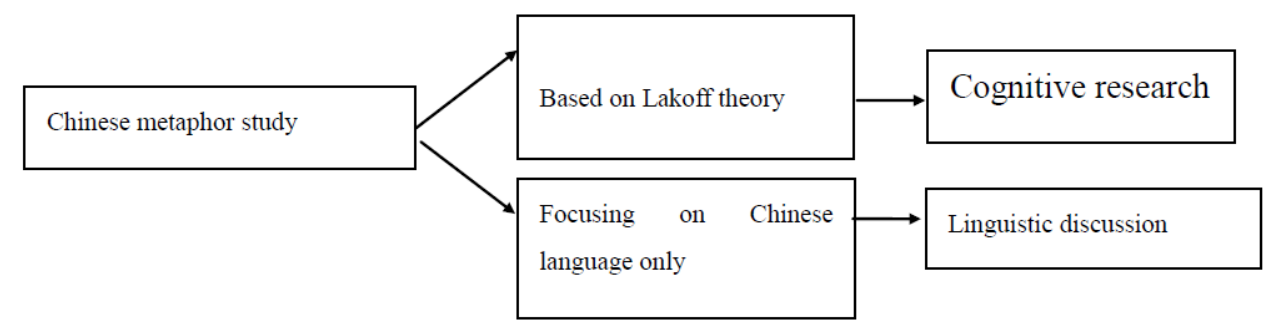

Figure 2 
The first group has a foreign language background and the area of their concern is also in this language. The second group is majored in Chinese language and their concern is also in Chinese language. Both groups should try to learn from each other and cooperate with each other. Only in this way, can the study on Chinese metaphor be more efficient. We try to make use of western contemporary theory of metaphor to analyze the phenomenon of metaphor in Chinese language. We hope this theory can present a totally new thought for Chinese metaphor study.

\section{REPRESENTATIONS OF SPATIAL CONCEPTS IN CHINESE}

Langman Modern English Dictionary defines 'Concept' as 'a general idea, thought of understanding'. According to Zheng (2001), concept reflects the basic characters of object or phenomenon, as the unit of thought. However, at linguistic level, concept is embodied by lexical expressions and sentences. According to Lakoff and Johnson, spatial concepts arise directly from our experience. They play an important role in our daily life. Limited by our body structure, biological organs and G-force, we get the concepts such as up/down, front/back and so on from our interaction with the world around us.

In English, when people talk of spatial system, they often mention the terms: up/down, front/back, high/low, in/out and etc. As for spatial system in Chinese language, Qi (1998) divides it into three subsystems, which include direction system, form system and seat system. These three systems are independent of each other as well as related to each other. Direction system is used to express direction. The words that are used to describe direction are called "directory words". Qi (1998) divides these words into three types according to different reference points they take. The first type is dong (east), nang (south), xi (west), bei (north). These words can denote direction without the help of reference point. To people who live on the earth, they know well their reference point. For instance, dong (east) is the direction where the sun rises. At linguistic level, such directory words still can be combined with affixes bian / mian / tou / fang, such as dong bian / dong mian /dong tou /dong fang, which also refer to the direction of east. The second type is qian/hou (front/back), shang/xia (up/down), zuo/you (left/right), which take one object as their reference points. For example, qian (front) refers to the direction one object faces. Shang (up) refers to the high part of one object. But for zuo/you (left/right), Qi argues that they are defined according to qian/hou (front/back). At this point, we think qian/hou (front/back), shang/xi (up/down), zuo/you (left/right) arise from our human body structure. We can take zuo/you (left/right) as an example to illustrate this. In the experience, people come to know the right hand is more agile than left one. They gradually distinguish them and gain the concepts of them. Then they use the concepts to describe other objects. For instance, we can say, "On the left of library is a hospital". For the third type, Qi says these directory words take one object added to the context as a reference point. Such directory words are li (in), wai (out), nei (in), zhong (middle), jian (middle) and pang (beside). These words can be combined with suffix tou/mian/bian, or with prefixes zhi/yi, for example: li tou (in), zhi jian (middle), yi nei (in).

For form system, Qi divides it into point, line, surface and volume. And then he discusses how the directory words are used within different forms, for example, "in" a form can be expressed by the directory words nei (in), zhong (middle), li (in), "out of" a form can be expressed by wai (out), jian (middle), pang (beside), dong (east), nan (south), xi (west), bei (north), qian/hou (front/back), zuo/you (left/right). In fact, form system has its own spatial concepts such as kuan/zhai (wide/narrow), cu/xi (wide/slender), hou/bao (thick/thin), qu/zhi (bent/straight), da/xiao (large/small) etc.

For seat form, Qi says one existing object must seat (occupy) some place. The object can be stationary or dynamic, which is relative according to its reference object. In modern Chinese, stationary seat of an object can be described by directory words. Dynamic seat of an object can be described by directory words as well as by verbs that have the meaning of "moving". For example: lai/qu (come/go), shang/xia (go up/ go down), jin/chu (come in/come out), hui (come back), qi (get up), guo (pass) and etc.

Macwhinney's (1999) also develops direction system into three types. He claims that the shape of human language is strongly influenced by the way in which perspective promoted the extraction of embodied meanings. He argues that spatio-temporal reference frames belong to such perspective-taking system. In fact, Qi's three types of "directory words" fall into Macwhinney's three alternative spatial frames. Qi's first type of “directory words” are Macwhinney's the environment-centered frame, which enforces a perspective based on fixed external landmarks, such as the position of a mountain range, the North star and so on. Qi's second type of “directory words"is Macwhinney's ego-centered frame, which encodes the perspective of the speaker. The terms that are grounded in the self's position and perspective include forward, backward, up, down, left and right. Qi's third type of "directory words" is Macwhinney's object-centered reference, which treats external objects as the centers of a complete ego perspective. In this sense, object-centered reference is best viewed as an extension of ego-centered reference grounded on a shift of perspective from ego to an external object.

On the other hand, apart from the spatial terms mentioned above, we believe there is another kind of spatial terms that come from human-body-part nouns by the means of metaphor. Human body plays an important role in understanding the world. As Kövecses (1999) suggests human biology influences the conceptualization of the different aspects of the concepts in different ways. Its influence seems to be stronger in ontology. Through investigating on the "container" metaphor of anger in English, Chinese, Japanese and Hungarian, Kövecses proposes that such cross-cultural similarity in conceptualization of anger should attribute to similarities in human body and its functioning in anger. In spatial conceptualization, human body senses the existing space and presents it to the brain, which forms some similar 
spatial representations in many languages. On the other hand, human also maps its own body parts onto other objects and forms the object-part nouns based on our body-part nouns. Through the study on Japanese language, Matsumoto (1999) finds such phenomenon existing in Japanese language. His explanation for this is that our body part has correspondent location on an object onto which human body is metaphorically mapped. Just because of this similarity of location, the object-part nouns that are based on human body-part nouns easily tend to become spatial terms. For example, human body part "foot" is expanded to the bottom of mountain and forms "the foot of maintain". In Chinese words, we can find a large number of such expressions such as: shan jiao (foot of mountain), shan tou (head of mountain), shan ji (backbone of mountain), shan yao (waist of maintain)... They're still many other Chinese lexical terms reflect such metaphorical thinking, for example:

for body part "mouth", there are ping zui (mouth of bottle), cha hu zui (mouth of teapot).

for "nose", men bi'er (nose of door), zhen bi'er (nose of needle)

for "teeth", ju chi (teeth of saw), shu chi (teeth of comb)

for "eye", quan yan (eye of spring), zhen yan'er (eye of needle)

for "leg", zuo tui (leg of table), ban deng tui (leg of bench)

In fact, such linguistic expressions can be found in many languages. In English, we speak of the hands of a clock, the teeth of a zipper, and the foot of the mountain. In Apache, the penchant for body-part metaphors carries over to describing the parts of an automobile. The tires are the feet of the car, the battery is its heart, and the headlights are the its eyes (Macwhinney, 1999). According to Kövecses (1999), such cross-cultural similarity ought to attribute to the similarity in human body structure. Mastumoto (1999) divides the similarity between body part and object part into three types. The first is similarity of location, such as body part "foot" similar to the bottom part of mountain. The second is similarity of shape, such as body part "teeth" similar to the correspondent part of a saw. The third is similarity of function, such as body part "leg" has the function similar to the supporting part of a table. Mastumoto (1999) also claims that the first type is much easier to develop into spatial nouns than last two types. As for this point, we agree on Mastumoto's arguments because similarity of location forms the location-based terms, which belong to "seat system" termed by Qi (1998). Based on the above discussion, we conclude the classification of Chinese spatial terms in the following figure:

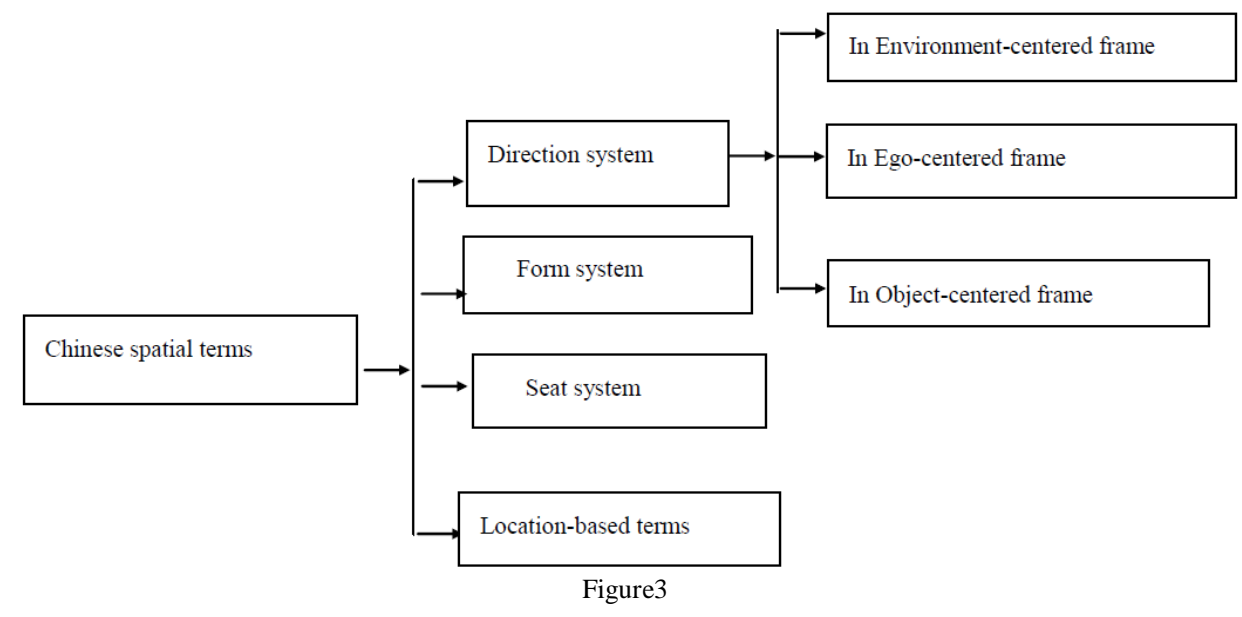

The spatial terms metaphorically adopted from location-based similarity with human body are less extended again. But this provides us a new way to study metaphor, that is, to study how our body parts are metaphorically extended and to what extent they are extended. For the goal of our study, we will leave this out.

\section{Metaphorical EXTEnsion OF Spatial Concepts in Chinese}

Based on the above discussion, we can conclude that most of spatial concepts arise directly from our interaction with the surroundings. Parts of them are developed through metaphor and have fewer tendencies to further metaphorically used on other terms. It is the spatial terms directly derived from our bodily experience that form the base on which we build up many other abstract concepts.

\section{A. Spatial Extension Represented in Chinese Lexis}

For spatial extension, Chinese lexical expressions provide amount of proof in their collocations. The compound words in Chinese language occupy an outstanding position. Their combinations present us much implication.

Let's first take shang/xia (up/down) as an example. Referring to leader and subordinate, Chinese has the following expressions, which involve shang/xia (up/down):

(2) Leader

a. shang ceng

subordinate

xia ceng 


$\begin{array}{lccc}\text { up level } & \text { down } & \text { level } \\ \text { b. shang } & \text { si } & \text { xia } & \text { shu } \\ \text { up manage } & \text { down } & \text { belong } \\ \text { c. shang mian } & \text { xia } & \text { mian } \\ \text { up suffix } & \text { down } & \text { suffix } \\ \text { d. shang tou } & \text { xia } & \text { tou } \\ \text { up head } & \text { down } & \text { head } \\ \text { e. shang ji } & \text { xia } & \text { ji } \\ \text { up grade } & \text { down } & \text { grade }\end{array}$

This is the extended expression of metaphors CONTROL IS UP and UNCONCTROL IS DOWN. Lakoff and Johnson (1980) have discussed such metaphors in English expressions. Since CONTROL IS UP, if people want more power to control, they should try to be up. So in Chinese, there are such expressions:

(3) Ta xiang jin le banfa wang shang pa

he think all ASP way to up climb

"He thinks all the ways to climb up."

(4) $\mathrm{Ni}$ hai you jihui sheng shang qu

you still have chance ascend up go

"You still have chance to be promoted."

(5) Ta you bei tiba shang qu le

he again BEI promote up go ASP

"He is promoted again."

$\mathrm{Up} /$ down can also be expanded to the concepts of GOODNESS/BADNESS. We can find their extension in following words:

(6) GOODNESS
a. shang deng
up class
b. shang ce
up strategy
c. shang pin
up quality

$\begin{array}{ll}\text { xia } & \text { deng } \\ \text { down } & \text { class } \\ \text { xia } & \text { ce } \\ \text { down } & \text { strategy } \\ \text { xia } & \text { pin } \\ \text { down } & \text { quality }\end{array}$

BADNESS

In Chinese, besides up/down, there are still other spatial terms metaphorically used in lexis, such as:

d. kuan hou

wide thick

Its metaphorical meaning is "honest and pure-hearted to people"

e. shen hou

deep thick

Its metaphorical meaning is "profound"

f. qian bo
shallow thin

Its metaphorical meaning is "lack of knowledge".

In modern Chinese, there are still many spatial terms used to modify some abstract concepts. For example, we say the following expressions frequently:

$\begin{array}{ccc}\text { (7) a. da/xiao wenti } & \text { da shiye } \\ \text { big/small problem } & \text { big business } \\ \text { b. xiao qingxu } & \text { da qihou } \\ \text { small mood } & \text { big climate } \\ \text { c. xiao piqi } & \text { cu } & \text { hua } \\ \text { small temper } & \text { thick words } \\ \text { d. cu huo } & \text { xiao shou wan'er } \\ \text { thick work } & \text { small wrist } \\ \text { e. xiao chongming } & \text { xiao jiqiao } \\ \text { small cleverness } & \text { small technique } \\ \text { f. xiao baogao } & \text { xiao xin yan'er } \\ \text { small report } & \text { small mind's eye }\end{array}$

The spatial words such as small/big, thick often are used to describe the form and seat of an object which exists in the space. In above examples, these spatial words are used to modify other abstract concepts such as temper, mood, cleverness, climate and so on. Such usages just reflect some metaphorical patterns. For instance, "big problem", "big business" and "big climate" right reflect the metaphorical pattern BIG IS IMPORTANT. This metaphor recurs across broad and unrelated languages. Grady (1997) finds this metaphorical pattern recurring in Zulu, Hawaiian, Turkish, Malay and Russian. In each of these languages, the term "big" which literally refers to the spatial form of a physical 
size can also refer metaphorically to degree of importance. This is a conceptualization with very wide cross-linguistic distribution. But how about the spatial term "small" in "small temper" and "small mood", "thick" in "thick talk" and "thick work"? Do their metaphorical patterns recur in many languages or only in Chinese? If not, why? For this is much complex to explain, we will talk it in latter research.

\section{B. Implication to Chinese Lexical Collocation}

According to the theory of conceptual metaphor, most of human concepts are organized through spatial metaphors. The above discussions give us some implication on how some Chinese compound words are formed. As we know linguistic system links with conceptual system. Metaphorical thinking process activates conceptual system, which can be embodied in linguistic system.

For a basic concept such as "UP" can metaphorically extend to a set of abstract concepts and forms the metaphors: HAPPY IS UP, CONTROL IS UP, and GOOD IS UP. Therefore, the concepts of HAPPY, CONTROL and GOOD can be united into one sort. Such consistency united by one spatial concept, makes these abstract concepts coherently collocated with the spatial term and form the collocations that are widely used in our linguistic expressions. For example, "good" plus "up" forms top-grade (shang hao), "low" plus "bad" forms inferior (di lie). On the other hand, one concept also can unite a set of spatial terms which are closely linked in spatial expressions. For example, "low status" unites "low" and "down" into di xia (low status), "profound" unites "high" and "deep" into gao shen (profound). There are still many such compounds in Chinese like kuanhou, shenhou, gaogao zai shang, zhi gao wu shang and so on.

\section{Body-Part Nouns Plus Spatial Terms}

In Chinese language, there still exists another phenomenon, that is, body-part nouns plus some spatial terms express not only location but also other abstract concepts such as time and status. For example, Chinese words like mu qian (in front of eyes), yen qian (in front of eyes), yan xia (under the eyes), yan di xia (under the eyes) often used to express time rather than location in their practical usage. In this phenomenon, we take time as a moving object: when an object comes to us, it is our eyes first catch it. So when the time comes to us and arrives in front of our eyes or under our eyes, it is present time. Body-part nouns plus spatial terms such as mian qian (in front of face), shen hou (behind one's body) also can be used to express time in their daily usage, for example:

(8) Mian qian de shi'er jiu gou nan chuli le face front DE matter just enough hard deal with ASP "It is hard to deal with current matter."

(9) Shen hou yao ban de shi'er hen zuo body behind to do DE things very many

"Later there are many things to do."

Here mian qian (in front of face) refers to present time, shen hou (behind one's body) refers to later time. For these two terms, we will explain their usages in next paper. Another body-part term "head" is located in the highest point in the whole body and is also the most important part. So the position above head must be higher and the position below head must be lower. Thus "head" plus spatial terms UP/DOWN is metaphorically mapped onto the social status: "up head" (shang tou) refers to superior; "down head" (xia tou) refers to subordinate. So Chinese has such expression:

(10) Shang tou zenyang yaoqiu, xia tou jiu zenyang qu duo up head how demand, down head PRT how go do "The subordinate do what the superior demand."

For another body part "hand" can manipulate many things, and spatial term "down" metaphorically means lower STATUAS, they combine together into " hand down" which in Chinese can refer to subordinate.

\section{CONCLUSION}

This paper mainly discusses present state of studies on spatial metaphors at home and abroad. Since language provides us with a good clue to discover the underlying metaphorical concept in our cognitive system, in this paper, we mainly focus on Chinese spatial concepts and try to explore how some spatial terms are metaphorically expanded to other concepts at linguistic level. Through the study on Chinese lexis, we find some spatial terms are metaphorically used in this field. From this aspect, we say metaphor is an important way to develop the lexis in Chinese language as well as to interpret its development.

\section{REFERENCES}

[1] Alverson, H. (1994). Semantic and experience: Universal metaphors of time in English, Mandarin, Hindi and Sesotho. Baltimore and London: The Johns Hopkins University Press.

[2] Coly, C. (2000). Metaphor and culture. In Robert A. Wilson and Frank C. Kell (eds.), The MIT Encyclopedia of the cognitive science. Shanghai: Shanghai Foreign Language Education Press, 221-230.

[3] Kövecses, Z. (1995). The "container" metaphor of anger in English, Chinese, Japanese and Hungarian. In Z. Radman (ed.), From a metaphorical point of view. Berlin/New York: Warter de Gruyter, 117-145. 
[4] Kövecses, Z. (2000a). The scope of metaphor. In Antonio Barcelona (ed.), Metaphor and metonymy at the crossroads. Berlin/New York: Mouton de Gruyter: 230-251.

[5] Kövecses, Z. (2000b). Metaphor and emotion. Cambridge: Cambridge University Press.

[6] Kövecses, Z. (1997). Does it constitute or reflect cultural models. In Gibbs and Steen (eds.), Metaphor in cognitive linguistics. Amsterdam: John Benjamins, 167-188.

[7] Lakoff, (1987). Women, fire, and dangerous things. Chicago: University of Chicago Press.

[8] Lakoff G. and Johnson. (1980). Metaphors we live by. Chicago: University of Chicago Press.

[9] Li, N. C. (1995). Ancestor-decedent and cultural-linguistic relativity. In M. Shibbatani and S.Thompson (eds.), The essays of semantics and pragmatics. Amsterdam: John Benjamins, 91-132.

[10] Macwhinney, B. (1999). The Emergence of language from embodiment. In B. Macwhinney (ed.), The emergence of language. London: Lawrence Erlbaum Associate, Inc., 213-250.

[11] Matsumoto, Y. (1999). Body-part nouns, object-part nouns and location-based object-part nouns. In B. A. Fox, D. Jurafsky \& L. A. Michaelis (eds.), Cognition and function in language. California: CSLI Publications, 126-134.

[12] Nuñez and Sweetser. (2001). Spatial embodiment of temporal metaphors in Aymara: blending source-domain gesture with speech. http://www.wam.umd.edu/ mturn/www/ICLC7.workshop.html (accessed 9/4/2003).

[13] Shinohara, K. (1998). Conceptual mappings from spatial motion to time: Analysis of English and Japanese. In C. I. Nehaniv (eds.), Computation for metaphors, analogy and agents. New York: Springer, 230-242.

[14] Yu, N. (1995). The contemporary theory of metaphor: A perspective from Chinese. Amsterdam: John Benjamins.

[15] Huang, P. (2001). Hanyu biyu yu minzu wenhua. Xiuci xuexi 3: 25-30.

[16] Hu, Youzhang. (2001). Biyu fawei. Xiuci xuexi 3: 31-32.

[17] Lan, Chun. (1999). Cong renzhi jiaodu kan hanyu de kongjian yinyu. Foreign language teaching and research 4: 7-15.

[18] Li, Chungxin. (2001). Han Tai chengyu yu ziren huanjing. Xiuci xuexi 6: 23-25

[19] Zhao, Yanfang. (2000). Renzhi yuyanxue de lilun ji xingcheng guocheng. Journal of foreign languages 1: 29-36.

[20] Zheng, Shupu. (2001). Cong "gainian” yici de shiyi shuoqi. Waiyu xuekan 1:13-16.

Jihua Fan was born in Henan, China in 1975. She received her M.A. degree in linguistics from Hunan University, China in 2003. She is currently an associate professor in the School of Foreign Languages, North China Institute, Beijing, China. Her research interests include cognitive linguistics and language teaching. 